\title{
Observing Quantum Trajectories: From Mott's Problem to Quantum Zeno Effect and Back
}

\author{
Maurice de Gosson* Basil Hiley $^{\dagger} \quad$ Eliahu Cohen ${ }^{\ddagger}$
}

November 13, 2018

\begin{abstract}
The experimental results of Kocsis et al., Mahler et al. and the proposed experiments of Morley et al. show that it is possible to construct "trajectories" in interference regions in a two-slit interferometer. These results call for a theoretical re-appraisal of the notion of a "quantum trajectory" first introduced by Dirac and in the present paper we re-examine this notion from the Bohm perspective based on Hamiltonian flows. In particular, we examine the short-time propagator and the role that the quantum potential plays in determining the form of these trajectories. These trajectories differ from those produced in a typical particle tracker and the key to this difference lies in the active suppression of the quantum potential necessary to produce Mott-type trajectories. We show, using a rigorous mathematical argument, how the active suppression of this potential arises. Finally we discuss in detail how this suppression also accounts for the quantum Zeno effect.
\end{abstract}

\section{Introduction}

There has been a revival of interest in the question of whether any meaning can be given to the notion of a particle trajectory in the quantum domain where field theory has already been so successful. However, the question originally raised by Kemmer [48] remains, namely, how do we discuss the

\footnotetext{
${ }^{*}$ University of Vienna, Faculty of Mathematics (NuHAG) Oskar-Morgenstern-Platz 1, 1090 Vienna.

${ }^{\dagger}$ Physics Department, University College, London, Gower Street, London WC1E 6BT and TPRU, Birkbeck, University of London, Malet Street, London WC1E 7HX.

${ }^{\ddagger}$ H.H. Wills Physics Laboratory, University of Bristol, Tyndall Avenue, Bristol, BS8 1TL, U.K.
} 
limiting process through which the particle is identified by the track it leaves in a tracking device like a bubble chamber. To construct a trajectory, we need the notion of a local momentum, a notion that has been assumed to be ruled out by the uncertainty principle. However, the uncertainty principle is about a simultaneous measurement of the position and momentum and cannot answer the question as to whether or not the quantum particle actually has a simultaneous value of its position and momentum. A further question remains, namely, that if a local momentum exists, how do we measure it?

Wiseman [67] was one of the first to point out that the weak value of the momentum operator is the local momentum or the Bohm momentum in the Bohm approach. (See also Hiley [39] for a wider perspective.) Duck, Stevenson and Sudarshan [15] have shown how weak values can be measured in what are called weak measurements, a result that immediately opens up the possibility of an experimental investigating the trajectories such as those calculated by Philippidis, Dewdney and Hiley [62] in the interference region of a two-slit interferometer. Indeed, the local momentum (essentially Poynting's vector [5]) of the electromagnetic field has already been measured in the experiments of Koscis et al. [50] and Mahler et al. [54] who used a quantum dot to generate a weak intensity field which was then passed through a two-slit system. Measurements of the local momentum were then used to construct what they called "average photon trajectories". In this sense they are returning to the notion of a "quantum trajectory" first introduced by Dirac 13 .

Although these flow lines have some resemblance to the trajectories of Philippidis et al. 62 referred to above, they cannot be compared directly because the latter are calculated using the Schrödinger equation, whereas photons are excitations of the electro-magnetic field. A treatment of the field approach from the Bohmian point of view has been given by Bohm, Hiley and Kaloyerou [7] and by Kaloyerou [45]. Thus it is not clear that photons can be considered to be travelling along trajectories. However Morley, Edmonds and Barker [58] are now carrying out a similar experiment using, instead, argon atoms with an aim to construct trajectories which can be directly compared with the theoretically predicted ones.

In light of this background, we explore the relation between the trajectories determined in a general interference region and the trajectories that are seen in a particle tracker more closely using the Hamiltonian flow method developed by de Gosson [21] which, in turn, clarifies the Bohm approach as discussed in Bohm and Hiley [8]. This latter approach centres on the real part of the Schrödinger equation under polar decomposition of the wave 
function, which takes the form

$$
\frac{\partial S^{\psi}}{\partial t}+\frac{\left(\nabla S^{\psi}\right)^{2}}{2 m}+V(x)+Q^{\Psi}(x, t)=0
$$

where $Q^{\Psi}(x, t)$ is the quantum potential defined by

$$
Q^{\Psi}(x, t)=-\frac{\hbar^{2}}{2 m} \frac{\nabla^{2} R^{\psi}(x, t)}{R^{\psi}(x, t)} .
$$

This equation, the quantum Hamilton-Jacobi equation, like its classical counterpart, enables us to calculate an ensemble of trajectories once we are given a solution of the Schrödinger equation for the experimental situation under investigation. Clearly, the key to the different forms of trajectories lies in the appearance of the quantum potential. We will show how this potential arises naturally in the method of Hamiltonian flows and study its behaviour in more detail.

As two of us [26] have already shown for the particular case of a point source, the quantum motion of the particle always reduces to the classical Hamiltonian trajectory for short times. Thus, in this case the nonappearance of the quantum potential will guarantee classical behaviour. In this paper we generalize our theory to the case of arbitrary initial conditions. In this way we provide a rigorous proof of how the Bohmian approach explains this phenomenon without appealing to any wavefunction collapse.

Our investigations show that the key to the appearance of the classical behaviour is the suppression of the quantum potential. Indeed we find that all quantum phenomena arise from the presence of this term in the real part of the Schrödinger equation. Therefore, its suppression will inhibit quantum transitions and this is what is required to explain the quantum Zeno effect.

A few words regarding the structure of this paper.

- In Section 2 we review the notion of short-time propagators for the Schrödinger equation using previous works of ours [21, 26, 28] and of Makri and Miller [52, 53] the main result is that one can replace the Van Vleck propagator - which is accurate to order $\Delta t^{2}$ - with what we call the Kerner-Sutcliffe propagator [49], which is much easier to use in explicit calculations since it does not require the evaluation of the exact action integral, but only of simply computable $\Delta t^{2}$ approximation thereof;

- In Section 3 we begin by revisiting the Bohmian theory of quantum motion, which reinstates the notion of trajectory in quantum mechanics; we emphasize the Hamiltonian nature of this motion, which has 
already been highlighted by Holland [41, 42. We thereafter utilize the results from Section 2 to show that to a very good approximation (i.e. to order $\left.\Delta t^{2}\right)$ these Bohmian trajectories are classical Hamiltonian trajectories.

- In Section 4 we employ our results to the study of the quantum Zeno effect for an arbitrary system of particles, using an extension to timedependent flows of the usual Lie-Trotter formula. We apply our results to the Mott problem.

We have highlighted the main mathematical results by dignifying them as "Theorems" rather than hiding them in a morass of calculations.

We will be working in position and momentum coordinates $x=\left(x_{1}, \ldots, x_{n}\right)$ and $p=\left(p_{1}, \ldots, p_{n}\right)$ referring to a N-body system; the corresponding phase space is thus $2 n$ dimensional. We recall Landau's "big $\mathrm{O}$ " notation: given two functions $f(t)$ and $g(t)$ the relation $f(t)-g(t)=\mathcal{O}\left(t^{k}\right)$ (for $t \rightarrow 0$ ) means that there exists a constant $C$ such that $|f(t)-g(t)| \leq C\left|t^{k}\right|$.

\section{Short-Time Action and Approximate Propaga- tors}

We begin by listing the assumptions we make on the quantum Hamiltonian. We thereafter shortly review the well-known approximation to the quantum mechanical propagator essentially due to Van Vleck. We then show that one can, with benefit, replace the latter with a simpler approximation, whose construction is based on exact asymptotic expressions of the action integral.

\subsection{Basic assumptions}

Let $\Psi$ be a solution of the multi-particle Schrödinger equation

$$
i \hbar \frac{\partial \Psi}{\partial t}(x, t)=\left[\sum_{j=1}^{n} \frac{-\hbar^{2}}{2 m_{j}} \frac{\partial^{2}}{\partial x_{j}^{2}}+V(x)\right] \Psi(x, t) .
$$

We will assume that given a square integrable initial value $\Psi\left(x, t_{0}\right)=\Psi_{0}(x)$ this solution exists and is unique. We moreover assume that if $\Psi_{0}(x)$ is infinitely differentiable in the position variables $x_{1}, \ldots, x_{n}$ so is $\Psi(x, t)$ for every time $t$ in some (usually small) interval $I_{T}=[-T, T]$. This assumption allows the quantum potential $Q(x, t)$ and its gradient (the quantum force, up to the sign) to be defined for $t \in I_{T}$. 
More precisely, we assume that the conditions for Stone's theorem that establishes a one-to-one correspondence between self-adjoint operators on the Hilbert space $L^{2}\left(\mathbb{R}^{n}\right)$ and one-parameter families of $\left(U_{t}\right)$ unitary operators are satisfied. This requires that the quantum Hamiltonian

$$
\widehat{H}=\sum_{j=1}^{n} \frac{-\hbar^{2}}{2 m_{j}} \frac{\partial^{2}}{\partial x_{j}^{2}}+V(x)
$$

be essentially self-adjoint on $L^{2}\left(\mathbb{R}^{n}\right)$, in which case the family $\left(U_{t}\right)$ is formally given by $U_{t}=e^{-i \widehat{H} t / \hbar}$ (see for instance Reed and Simon [63]). It is well-known (following Kato [46]) that this is the case for the majority of physically interesting potentials $V(x)$, including Coulomb-type potentials of the form $r^{-m}$ for $m<3 / 2$. The essential point in Kato's proof is that we should have

$$
V \Psi \in L^{2}\left(\mathbb{R}^{n}\right) \text { for all } \Psi(x)=P(x) e^{-|x|^{2} / 2}
$$

where $P(x)=P\left(x_{1}, \ldots, x_{n}\right)$ is a polynomial in position coordinates. Notice that Kato's condition also trivially holds when the Hamiltonian function is a quadratic polynomial in the position variables (one can produce in this case explicit formulas for $U_{t}$ using the metaplectic representation [21, 22]). More generally (44) will hold for all potential functions that are continuously differentiable to all orders in the $x_{1}, \ldots, x_{n}$ variables, and for which there exists, for every multi-index $\alpha \in \mathbb{N}^{n}$ real constants $C_{\alpha}>0$ and $m_{\alpha}$ such that

$$
\left|\partial_{x}^{\alpha} V(x)\right| \leq C_{\alpha}(1+|x|)^{m_{\alpha}} .
$$

Condition (5) can be relaxed in various ways; for instance one can assume that the potential is only continuously differentiable up to a finite order ensuring the existence of the quantum potential and its derivatives.

Defining for two arbitrary times $t, t^{\prime} \in I_{T}$ the operator $U_{t, t^{\prime}}=U_{t} U_{-t^{\prime}}$ on $L^{2}\left(\mathbb{R}^{n}\right)$ we have:

(1) $U_{t, t^{\prime}} U_{t^{\prime}, t^{\prime \prime}}=U_{t, t^{\prime \prime}}$ and $U_{t, t}=I_{\mathrm{d}}$ for all $t, t^{\prime}, t^{\prime \prime} \in I_{T}$;

(2) $U_{t, t^{\prime}}$ is strongly continuous in $L^{2}\left(\mathbb{R}^{n}\right)$ with respect to $t, t^{\prime}$;

(3) $\Psi(x, t)=U_{t, t_{0}} \Psi_{0}(x)$ is a solution of (2) for every $\Psi_{0} \in L^{2}\left(\mathbb{R}^{n}\right)$;

(4) $U_{t, t^{\prime}}$ maps $L^{2}\left(\mathbb{R}^{n}\right) \cap C^{\infty}\left(\mathbb{R}^{n}\right)$ into itself for all $t, t^{\prime} \in I_{T}$.

Notice that it follows by induction from the groupoid property $U_{t, t^{\prime}} U_{t^{\prime}, t^{\prime \prime}}=$ $U_{t, t^{\prime \prime}}$ that for every integer $N \geq 1$

$$
U_{t, t_{0}}=U_{t, t_{N-1}} U_{t_{N-1}, t_{N-2}} \cdots U_{t_{1}, t_{0}}
$$


hence we also have

$$
U_{t, t_{0}}=\lim _{N \rightarrow \infty}\left(U_{t, t_{N-1}} U_{t_{N-1}, t_{N-2}} \cdots U_{t_{1}, t_{0}}\right)
$$

and this identity also holds if we replace in the right-hand side the operators $U_{t_{j+1}, t_{j}}$ with second-order approximations $\bar{U}_{t_{j+1}, t_{j}}=U_{t_{j+1}, t_{j}}+\mathcal{O}\left(\left(t_{j+1}-\right.\right.$ $\left.\left.t_{j}\right)^{2}\right)$ :

$$
U_{t, t_{0}}=\lim _{N \rightarrow \infty}\left(\bar{U}_{t, t_{N-1}} U_{t_{N-1}, t_{N-2}} \cdots \bar{U}_{t_{1}, t_{0}}\right) .
$$

This follows from the Lie-Trotter-Kato theory (Chorin et al. [10]), and will be used in Section 2.3 (formula (27).

It follows from Schwartz's kernel theorem that the conditions (1)-(3) ensure the existence of a distribution $K=K\left(x, x^{\prime}, t, t^{\prime}\right)$ (the "propagator") such that

$$
\Psi(x, t)=\int K\left(x, x^{\prime}, t, t^{\prime}\right) \Psi\left(x^{\prime}, t^{\prime}\right) d^{n} x^{\prime}
$$

the property $U_{t, t}=I_{\mathrm{d}}$ is equivalent to $K\left(x, x^{\prime}, t, t\right)=\delta\left(x-x^{\prime}\right)$; it follows that for fixed $x^{\prime}$ and $t^{\prime}$ the function $K\left(x, x^{\prime}, t, t^{\prime}\right)$ is a solution of Schrödinger equation (2) with initial condition $\Psi^{\prime}\left(x, t^{\prime}\right)=\delta\left(x-x^{\prime}\right)$. Physically, it represents the wavefunction of a point source located at $x^{\prime}$ at the initial time $t^{\prime}$.

Here is a (very) short guide to the existing literature complementing the discussion above: Doi [14] studies the regularity problem of the solutions to (2) from the point of view of energy estimates. Yajima [70] states some precise condition, weaker than (5), guaranteeing the existence of the propagator for potentials generalising the Coulomb case. Leforestier et al. [51] discuss in depth various approximate propagation schemes for very general potentials which can easily be numerically implemented.

\subsection{The Van Vleck Propagator}

We will denote the initial time by $t_{0}$ and write $\Delta t=t-t_{0}$.

Consider the Schrödinger equation (2) with initial value $\Psi\left(x, t_{0}\right)=\Psi_{0}(x)$; we assume that $\Psi_{0}(x)$ is infinitely differentiable and belongs to $L^{2}\left(\mathbb{R}^{n}\right)$. We will denote by

$$
K\left(x, x_{0}, t, t_{0}\right)=\left\langle x\left|U_{t, t_{0}}\right| x_{0}\right\rangle=\left\langle x\left|e^{-\frac{i}{\hbar} \widehat{H}\left(t-t_{0}\right)}\right| x_{0}\right\rangle
$$

the corresponding exact propagator. The solution of (2) at time $t$ is thus given by the formula

$$
\Psi(x, t)=\int K\left(x, x_{0}, t, t_{0}\right) \Psi_{0}\left(x_{0}\right) d^{n} x_{0} .
$$


The condition $\lim _{t \rightarrow t_{0}} \Psi(x, t)=\Psi_{0}(x)$ implies that we must have

$$
\lim _{t \rightarrow 0} K\left(x, x_{0}, t, t_{0}\right)=\delta\left(x-x_{0}\right)
$$

since $K$ satisfies Schrödinger's equation for fixed $x_{0}$ and $t_{0}$. This can be viewed as the wavefunction corresponding to a point source located at $x_{0}$ at time $t=t_{0}$.

It is well-known (see Gutzwiller's discussion in [31], $\S 1.6$ and 12.5; also [21, [56, 65]) that for short times an approximate propagator is given by Van Vleck's formula

$$
\widetilde{K}\left(x, x_{0}, t, t_{0}\right)=\left(\frac{1}{2 \pi i \hbar}\right)^{n / 2} \sqrt{\rho\left(x, x_{0}, t, t_{0}\right)} e^{\frac{i}{\hbar} S\left(x, x_{0}, t, t_{0}\right)}
$$

where the function

$$
S\left(x, x_{0}, t, t_{0}\right)=\int_{t_{0}}^{t}\left(\sum_{j=1}^{n} \frac{1}{2} m_{j} \dot{x}_{j}(s)^{2}-V(x(s))\right) d s
$$

is the action along the classical trajectory leading from $x_{0}$ at time $t=t_{0}$ to $x$ at time $t$ (there is no sum over different classical trajectories because only one trajectory contributes in the limit $t \rightarrow t_{0}$ ), and

$$
\rho\left(x, x_{0}, t, t_{0}\right)=\operatorname{det}\left(-\frac{\partial^{2} S\left(x, x_{0}, t, t_{0}\right)}{\partial x_{j} \partial x_{0, k}}\right)_{1 \leq j, k \leq n}
$$

is the Van Vleck density of trajectories; the argument of the square root in (11) is chosen so that the initial condition (10) is satisfied. A caveat: the Van Vleck propagator is frequently used in semiclassical mechanics [31], it is however not a "semiclassical" object per se: it is genuinely an approximation to the exact propagator for small values of $\Delta t=t-t_{0}$ - not just in the limit $\hbar \rightarrow 0$. In fact (see for instance [21], Lemma 241), setting

$$
\widetilde{\Psi}(x, t)=\int \widetilde{K}\left(x, x_{0}, t, t_{0}\right) \Psi_{0}\left(x_{0}\right) d^{n} x_{0}
$$

we have

$$
\Psi\left(x, t_{0}+\Delta t\right)-\widetilde{\Psi}\left(x, t_{0}+\Delta t\right)=\mathcal{O}\left(\Delta t^{2}\right)
$$

and hence, in particular,

$$
K\left(x, x_{0}, t_{0}+\Delta t, t_{0}\right)-\widetilde{K}\left(x, x_{0}, t_{0}+\Delta t, t_{0}\right)=\mathcal{O}\left(\Delta t^{2}\right)
$$

for small values of $\Delta t=t-t_{0}$. 


\subsection{The Kerner and Sutcliffe propagator}

While the van Vleck propagator (11) is accurate to order $\Delta t^{2}$, it is, however, difficult to calculate in practice since it involves the action integral (12), whose determination in principle requires the resolution of Hamilton's equations for the classical Hamiltonian $H$, which is, outside a few trivial cases, a quite cumbersome task. We therefore construct another simpler approximation to same order $\Delta t^{2}$ of the exact propagator. For this purpose we begin by remarking that the exact action integral (12) is approximated by the function

$$
\bar{S}\left(x, x_{0}, t, t_{0}\right)=\sum_{j=1}^{n} m_{j} \frac{\left(x_{j}-x_{0, j}\right)^{2}}{2\left(t-t_{0}\right)}-\bar{V}\left(x, x_{0}\right)\left(t-t_{0}\right)
$$

where $\bar{V}\left(x, x_{0}\right)$ is the average of the potential $V$ along the line segment $\left[x_{0}, x\right]$ :

$$
\bar{V}\left(x, x_{0}\right)=\int_{0}^{1} V\left(\tau x+(1-\tau) x_{0}\right) d \tau .
$$

In fact, one proves [21, 52, 53] that for short times $\Delta t$ we have

$$
S\left(x, x_{0}, t_{0}+\Delta t, t_{0}\right)-\bar{S}\left(x, x_{0}, t_{0}+\Delta t, t_{0}\right)=\mathcal{O}\left(\Delta t^{2}\right) .
$$

For this, it suffices to remark that the function $S=S\left(x, x_{0}, t, t_{0}\right)$ satisfies the Hamilton-Jacobi equation

$$
\frac{\partial S}{\partial t}+\sum_{j=1}^{n} \frac{1}{2 m_{j}}\left(\frac{\partial S}{\partial x_{j}}\right)^{2}+V(x)=0
$$

and one thereafter looks for an asymptotic solution

$$
S\left(x, x_{0}, t, t_{0}\right)=\frac{1}{t-t_{0}} S_{0}\left(x, x_{0}\right)+S_{1}\left(x, x_{0}\right)\left(t-t_{0}\right)+\mathcal{O}\left(\left(t-t_{0}\right)^{2}\right) .
$$

Insertion in (19) then leads to

$$
S_{0}\left(x, x_{0}\right)=\sum_{j=1}^{n} m_{j} \frac{\left(x_{j}-x_{0, j}\right)^{2}}{2}
$$

(which is the free generating function) and $S_{1}\left(x, x_{0}\right)=-\bar{V}\left(x, x_{0}\right)$ hence (16). We emphasize that this approximation to the action is very different from the "midpoint-rules" commonly used in the theory of the Feynman path integral 
[65, which only yield, at best, $\mathcal{O}(\Delta t)$ approximations (see the discussions in $[21,26,28,52,53])$. We moreover point out that this procedure can be used to obtain approximations of $S\left(x, x_{0}, t, t_{0}\right)$ to arbitrary order $\mathcal{O}\left(\Delta t^{N}\right)$ by adding terms $S_{j}\left(x, x_{0}\right)\left(t-t_{0}\right)^{j}(j=2, \ldots, N)$ to the asymptotic sum (20) and determining the functions $S_{j}\left(x, x_{0}\right)$ by successive quadratures (see Makri and Miller [52, 53] for explicit calculations).

The next step consists of setting

$$
\bar{H}\left(x, x_{0}\right)=\sum_{j=1}^{n} \frac{p_{j}^{2}}{2 m_{j}}+\bar{V}\left(x, x_{0}\right)
$$

where $\bar{V}\left(x, x_{0}\right)$ is the averaged potential (17). One then sets

$$
\bar{K}\left(x, x_{0}, t, t_{0}\right)=\left(\frac{1}{2 \pi \hbar}\right)^{n} \int e^{\frac{i}{\hbar}\left(p\left(x-x_{0}\right)-\bar{H}\left(x, x_{0}, p\right)\left(t-t_{0}\right)\right)} d^{n} p ;
$$

using the theory of Fresnel integrals, it is easy to show after a few calculations that

$$
\bar{K}\left(x, x_{0}, t, t_{0}\right)=\left(\frac{m_{1} \cdots m_{n}}{2 \pi i \hbar\left(t-t_{0}\right)}\right)^{n / 2} e^{\frac{i}{\hbar} \bar{S}\left(x, x_{0}, t, t_{0}\right)}
$$

where $\bar{S}\left(x, x_{0}, t\right)$ is given by (16), and the argument of the square root is chosen so that $\lim _{t \rightarrow 0} \bar{K}\left(x, x_{0}, t\right)=\delta\left(x-x_{0}\right)$. Using the short-time estimate (18) one then shows that

$$
K\left(x, x_{0}, t_{0}+\Delta t, t_{0}\right)-\bar{K}\left(x, x_{0}, t_{0}+\Delta t, t_{0}\right)=\mathcal{O}\left(\Delta t^{2}\right)
$$

for small $\Delta t$. Defining the approximate wavefunction

$$
\bar{\Psi}(x, t)=\int \bar{K}\left(x, x_{0}, t, t_{0}\right) \Psi_{0}\left(x_{0}\right) d^{n} x_{0}
$$

it follows that

$$
\bar{\Psi}\left(x, t_{0}+\Delta t\right)-\Psi\left(x, t_{0}+\Delta t\right)=\mathcal{O}\left(\Delta t^{2}\right)
$$

for small values of $\Delta t$. The approximations above are, in fact, of order $O\left(\Delta t^{2}\right)$; in view of formula (15), they are thus as good, for all practical purposes, as the Van Vleck approximation $\widetilde{K}\left(x, x_{0}, t\right)$ considered in the previous section; the propagator $\bar{K}\left(x, x_{0}, t, t_{0}\right)$ can, however, be easily calculated since it only involves the determination of the integral (17) giving the average value of the potential along the straight line leading from $x_{0}$ to $x$. 
We notice that the propagator $\bar{K}\left(x, x_{0}, t, t_{0}\right)$ was postulated by Garrod [19], as well as Kerner and Sutcliffe [49], though they failed to prove the estimates (25) and (26). For details we refer to de Gosson's recent works [24, 25].

We mention that the fact that $\bar{K}\left(x, x_{0}, t, t_{0}\right)$ is an $\mathcal{O}\left(\Delta t^{2}\right)$ approximation to the exact propagator implies that we can construct the exact evolution operator for Schrödinger's equation by a "time-slicing" process [21]. In fact, defining the unitary operator $U_{t, t_{0}}$ by

$$
U_{t, t_{0}} \Psi_{0}(x)=\int K\left(x, x_{0}, t, t_{0}\right) \Psi_{0}\left(x_{0}\right) d^{n} x_{0}
$$

(it is the exact evolution operator for the Schrödinger equation) and its (non-unitary) approximation

$$
\bar{U}_{t, t_{0}} \Psi_{0}(x)=\int \bar{K}\left(x, x_{0}, t, t_{0}\right) \Psi_{0}\left(x_{0}\right) d^{n} x_{0}
$$

we have, in view of the estimate (26) and the discussion in Section 2.1),

$$
U_{t, t_{0}}=\lim _{N \rightarrow \infty}\left(\bar{U}_{t, t_{N-1}} \bar{U}_{t_{N-1}, t_{N-2}} \cdots \bar{U}_{t_{1}, t_{0}}\right)
$$

where $t_{0}<t_{1}<t_{2}<\cdots<t_{N-1}<t$ is a subdivision of the interval $\left[t_{0}, t\right]$ such that $\left|t_{j+1}-t_{j}\right|<\left(t-t_{0}\right) / N$. This can be proven using telescopic sums and the Lie-Trotter-Kato formula (see the Appendix B of [21], or, for more details, Chorin et al.. [10] and Nelson [60]). As Makri and Miller [52, 53, have shown, the speed of convergence of formula (27) is far superior to that obtained by using the usual Feynman-type approaches and is particularly well adapted to numerical calculations using Monte-Carlo methods.

\subsection{A simple example}

Let $H$ be the Hamiltonian function of the one-dimensional harmonic oscillator

$$
H=\frac{1}{2 m}\left(p^{2}+m^{2} \omega^{2} x^{2}\right)
$$

choosing $t_{0}=0$ the action integral $S$ is here given by

$$
S\left(x, x_{0}, t\right)=\frac{1}{2 \sin \omega t}\left(\left(x^{2}+x_{0}^{2}\right) \cos \omega t-2 x x_{0}\right)
$$

and the exact propagator for the corresponding Schrödinger equation

$$
i \hbar \frac{\partial \Psi}{\partial t}(x)=\left[-\frac{\hbar^{2}}{2 m} \frac{\partial^{2}}{\partial x^{2}}+\frac{m \omega^{2}}{2} x^{2}\right] \Psi(x)
$$


is the Mehler kernel

$$
K\left(x, x_{0}, t\right)=\left(\frac{m \omega}{2 \pi i \hbar \sin \omega t}\right)^{1 / 2} \exp \left[\frac{i}{2 \hbar \sin \omega t}\left(\left(x^{2}+x_{0}^{2}\right) \cos \omega t-2 x x_{0}\right)\right]
$$

where the argument of the first factor in the left-hand side is chosen so that $\lim _{t \rightarrow 0} K\left(x, x_{0}, t\right)=\delta\left(x-x_{0}\right)$. Using second order Taylor expansions of $\sin \omega t$ and $\cos \omega t$ at the origin one approximates $S\left(x, x_{0}, t\right)$ by

$$
\bar{S}\left(x, x_{0}, t\right)=m \frac{\left(x-x_{0}\right)^{2}}{2 t}-\frac{m \omega^{2}}{2}\left(x^{2}+x x_{0}+x_{0}^{2}\right) t
$$

which is precisely the value one obtains using formulas (16) and (17) since we have

$$
\begin{aligned}
\bar{V}\left(x, x_{0}\right) & =\frac{m \omega^{2}}{2} \int_{0}^{1}\left(\tau x+(1-\tau) x_{0}\right)^{2} d \tau \\
& =\frac{m \omega^{2}}{2}\left(x^{2}+x x_{0}+x_{0}^{2}\right) .
\end{aligned}
$$

The corresponding Kerner-Sutcliffe propagator is thus given by

$$
\bar{K}\left(x, x_{0}, t\right)=\left(\frac{m}{2 \pi i \hbar t}\right)^{1 / 2} \exp \left[\frac{i}{\hbar}\left(m \frac{\left(x-x_{0}\right)^{2}}{2 t}-\frac{m \omega^{2}}{2}\left(x^{2}+x x_{0}+x_{0}^{2}\right)\right)\right]
$$

which is in agreement with formula (23).

\section{Bohmian Trajectories}

\subsection{Quantum motion}

The idea lying behind the Bohmian approach (Bohm and Hiley [8], Hiley [36], Hiley and collaborators [37, 38], Holland [40, Wyatt [68]) is the following: let $\Psi$ be the wavefunction solution of Schrödinger's equation (2); we assume we assume that $\Psi(x)$ is infinitely differentiable. Writing $\Psi$ in polar form $\sqrt{\rho} e^{i S / \hbar}$, this equation, as we have already indicated, is equivalent, for $\rho(x, t) \neq 0$, to the coupled system of partial differential equations:

$$
\frac{\partial S}{\partial t}+\sum_{j=1}^{n} \frac{1}{2 m_{j}}\left(\frac{\partial S}{\partial x_{j}}\right)^{2}+V+Q^{\Psi}=0
$$

where the term

$$
Q^{\Psi}=-\sum_{j=1}^{n} \frac{\hbar^{2}}{2 m_{j}} \frac{1}{\sqrt{\rho}} \frac{\partial^{2} \sqrt{\rho}}{\partial x_{j}^{2}}
$$


is Bohm's quantum potential; equivalently

$$
Q^{\Psi}=-\sum_{j=1}^{n} \frac{\hbar^{2}}{4 m_{j}}\left(\frac{1}{\rho} \frac{\partial^{2} \rho}{\partial x_{j}^{2}}-\frac{1}{2 \rho^{2}}\left(\frac{\partial \rho}{\partial x_{j}}\right)^{2}\right) .
$$

Notice that the quantum potential is usually time-dependent: $Q^{\Psi}=Q^{\Psi}(x, t)$ since it is expressed in terms of the time-dependent function $\rho(x, t)$. We will assume that $\rho_{0}(x)=\rho\left(x, t_{0}\right)$ does not vanish, that is $\rho_{0}(x)>0$ for all $x$ in some domain $\Omega$. It follows by continuity that we will also have $\rho(x, t)>0$ provided that $\Delta t=t-t_{0}$ is small enough.

Equation (30) is, mathematically speaking, just the Hamilton-Jacobi equation (or Euler equation in fluid dynamics) for $H^{\Psi}=H+Q^{\Psi}$, and

$$
\frac{\partial \rho}{\partial t}+\sum_{j=1}^{n} \frac{1}{m_{j}} \frac{\partial}{\partial x_{j}}\left(\rho \frac{\partial S}{\partial x_{j}}\right)=0
$$

is the equation of continuity familiar again from hydrodynamics [11; it ensures the conservation of probability (see the recent work by Heifetz and Cohen [33. for a detailed discussion of these equations in terms of the Madelung fluid). In Bohm's theory of motion the trajectory of the system of particles is determined by the equations

$$
m_{j} \dot{x}_{j}^{\Psi}=\frac{\partial S}{\partial x_{j}}\left(x^{\Psi}, t\right), x^{\Psi}\left(t_{0}\right)=x_{0}
$$

where $x_{0}=\left(x_{0,1}, \ldots, x_{0, n}\right)$ is the initial position.

At this point we wish to make the following remark: the simple derivation of equations (30) to (33) obscures a deeper mathematical relation between the Hilbert space formalism of quantum mechanics and the Hamiltonian flows of classical mechanics. This exact relationship has been derived in very general terms by de Gosson and Hiley [27]; for a detailed mathematical study of that correspondence see the recent paper by de Gosson [23]. There is a one-to-one and onto correspondence between Hamiltonian flows generated by a Hamiltonian $H$ and the strongly continuous unitary oneparameter evolution groups $\left(U_{t}\right)$ satisfying Schrödinger's equation with the Hamiltonian operator $H\left(x,-i \hbar \nabla_{x}\right)$. This relation exploits the metaplectic representation of the underlying symplectic structure [22, 23] of classical mechanics in its Hamiltonian formulation. It is the metaplectic structure that gives rise to the quantum properties. Since the classical and quantum motions are related but different, it was proposed in de Gosson [21] to call the object that obeys the Bohmian law of motion (34) a metatron. 


\subsection{The Hamiltonian character of Bohmian trajectories}

Let $p_{0}=\left(p_{1,0}, \ldots, p_{n, 0}\right)$ be an arbitrary momentum vector, and set

$$
p_{0}=-\nabla_{x_{0}} S\left(x, x_{0} ; t, t_{0}\right) .
$$

Viewing $x, x_{0}$ as independent variables, the action $S\left(x, x_{0} ; t, t_{0}\right)$ is a generating function for $t-t_{0} \neq 0$ sufficiently small [3, 20, 21, 22]; in particular the Hessian matrix

$$
S_{x, x_{0}}^{\prime \prime}=\left(\frac{\partial^{2} S}{\partial x_{j} \partial x_{0, k}}\right)_{1 \leq j, k \leq n}
$$

is invertible: $\operatorname{det} S_{x, x_{0}}^{\prime \prime} \neq 0$. It follows from the implicit function theorem that equation (35) determines a function $x=x^{\Psi}(t)$ (depending on $x_{0}$ and $t_{0}$ viewed as parameters), defined by

$$
p_{0}=-\nabla_{x_{0}} S\left(x^{\Psi}(t), x_{0} ; t, t_{0}\right) .
$$

Setting

$$
p^{\Psi}(t)=\nabla_{x} S\left(x^{\Psi}(t), x_{0} ; t, t_{0}\right)
$$

we claim that the functions $x^{\Psi}(t)$ and $p^{\Psi}(t)$ thus defined are solutions of the Hamilton equations

$$
\left.\dot{x}^{\Psi}(t)=\nabla_{p} H^{\Psi}\left(x^{\Psi}(t), p^{\Psi}(t), t\right), \quad \dot{p}^{\Psi}(t)=-\nabla_{x} H^{\Psi}\left(x^{\Psi}(t), p^{\Psi}(t), t\right)\right)
$$

with initial conditions $x^{\Psi}\left(t_{0}\right)=x_{0}, p^{\Psi}\left(t_{0}\right)=p_{0}$; here $H^{\Psi}=H+Q^{\Psi}$ where $Q^{\Psi}$ is the quantum potential (31). We are going to use classical HamiltonJacobi theory. For notational simplicity we assume here that $n=1$; the generalization to the case of an arbitrary number $n$ of degrees of freedom is straightforward. The quantum Hamilton-Jacobi equation

$$
\frac{\partial S}{\partial t}+H^{\Psi}\left(x, \frac{\partial S}{\partial x}, t\right)=0
$$

can be treated mathematically using Hamilton-Jacobi theory. Differentiating both sides of (40) with respect to $p$ and using the chain rule, we get

$$
\frac{\partial^{2} S}{\partial x_{0} \partial t}+\frac{\partial H^{\Psi}}{\partial p} \frac{\partial^{2} S}{\partial x_{0} \partial x}=0
$$

and differentiating equation (37) with respect to time $t$ yields

$$
\frac{\partial^{2} S}{\partial x_{0} \partial t}+\frac{\partial^{2} S}{\partial x \partial x_{0}} \dot{x}^{\Psi}=0 .
$$


Subtracting (42) from (41) we get

$$
\frac{\partial^{2} S}{\partial x \partial x_{0}}\left(\frac{\partial H^{\Psi}}{\partial p}-\dot{x}^{\Psi}\right)=0
$$

which produces the first Hamilton equation (39) since it is assumed that we have $\partial^{2} S / \partial x \partial x_{0} \neq 0$ (condition (36) in the case $n=1$ ). Let us next show that the second Hamilton equation (39) is satisfied as well. For this we differentiate the quantum Hamilton-Jacobi equation (40) with respect to $x$, which yields

$$
\frac{\partial^{2} S}{\partial x \partial t}+\frac{\partial H^{\Psi}}{\partial x}+\frac{\partial H^{\Psi}}{\partial p} \frac{\partial^{2} S}{\partial x^{2}}=0
$$

Differentiating the equality (38) with respect to $t$ we get

$$
\frac{\partial^{2} S}{\partial t \partial x}=-\dot{p}^{\Psi}(t)-\frac{\partial^{2} S}{\partial x^{2}} \dot{x}^{\Psi}
$$

and hence equation (43) can be rewritten as

$$
-\dot{p}^{\Psi}-\frac{\partial^{2} S}{\partial x^{2}} \dot{x}^{\Psi}+\frac{\partial H^{\Psi}}{\partial x}+\frac{\partial H^{\Psi}}{\partial p} \frac{\partial^{2} S}{\partial x^{2}}=0 .
$$

Taking into account the relation $\dot{x}^{\Psi}=\partial H^{\Psi} / \partial p$ established above we have

$$
-\dot{p}^{\Psi}-\frac{\partial H^{\Psi}}{\partial x}=0
$$

which is precisely the second Hamilton equation (39). There remains to show that the initial value conditions $x^{\Psi}\left(t_{0}\right)=x_{0}$ and $p^{\Psi}\left(t_{0}\right)=p_{0}$ are satisfied. Recalling that $x^{\Psi}(t)$ is defined by the implicit equation

$$
p_{0}=-\frac{\partial S}{\partial x_{0}}\left(x^{\Psi}(t), x_{0} ; t, t_{0}\right)
$$

(equation (37)) the formulas (18), (16) imply that

$$
p_{0}=\frac{m\left(x^{\Psi}(t)-x_{0}\right)}{t-t_{0}}-\frac{\partial \bar{V}}{\partial x_{0}}\left(x^{\Psi}(t), x_{0}\right)\left(t-t_{0}\right)+\mathcal{O}\left(\left(t-t_{0}\right)^{2}\right) ;
$$

taking the limit $t \rightarrow t_{0}$ and noting that

$$
\frac{\partial \bar{V}}{\partial x}\left(x_{0}\right)=\int_{0}^{1} \tau \frac{\partial V}{\partial x}\left(\tau x_{0}+(1-\tau) x_{0}\right) d \tau=\frac{1}{2} \frac{\partial V}{\partial x}\left(x_{0}\right)
$$


we get both $x^{\Psi}\left(t_{0}\right)=x_{0}$ and $p_{0}=m \dot{x}^{\Psi}\left(t_{0}\right)=p^{\Psi}\left(t_{0}\right)$.

We refer to the papers by Holland [41, 42] for a thorough discussion of the interpretation of Bohmian trajectories from the Hamiltonian point of view.

To complete our discussion, we make the two following observations:

- Even when the Hamiltonian function $H$ does not depend explicitly on time, the function $H^{\Psi}=H+Q^{\Psi}$ is usually time-dependent (because the quantum potential generally is), so the flow $\left(f_{t}^{\Psi}\right)$ it determines does not inherit the usual group property $f_{t} f_{t^{\prime}}=f_{t+t^{\prime}}$ of the flow determined by the classical Hamiltonian $H$. One has to use instead the "time-dependent flow" $\left(f_{t, t^{\prime}}^{\Psi}\right)$, defined by $f_{t, t^{\prime}}^{\Psi}=f_{t}^{\Psi}\left(f_{t^{\prime}}^{\Psi}\right)^{-1}$, which

has a groupoid property [1, 2] in the sense that $f_{t, t^{\prime}}^{\Psi} f_{t^{\prime}, t^{\prime \prime}}^{\Psi}=f_{t, t^{\prime \prime}}^{\Psi}$ (the Chapman-Kolmogorov law, which expresses causality);

- The time-dependent flow $\left(f_{t, t^{\prime}}^{\Psi}\right)$ consists of canonical transformations; that is, the Jacobian matrix of $f_{t, t^{\prime}}^{\Psi}$ calculated at any point $(x, p)$, where it is defined by a symplectic matrix. This is an immediate consequence of the fact discussed above, namely, that the flow determined by any Hamiltonian function has this property [3, 20, 21, 22]. There is thus a one-to-one correspondence between the quantum flow $\left(f_{t}^{\Psi}\right)$ and the classical Hamiltonian flow $\left(f_{t}\right)$ (see de Gosson [23] for a detailed study of this correspondence).

\subsection{Short-time solutions}

We have seen that the Bohmian trajectory for a particle initially sharply localized at a point $x_{0}$ is Hamiltonian, and in fact governed by the Hamilton equations (39):

$$
\dot{x}=\nabla_{p} H^{\Psi}(x, p, t), \dot{p}=-\nabla_{x} H^{\Psi}(x, p, t) .
$$

corresponding to the $\Psi$-dependent Hamiltonian function $H^{\Psi}=H+Q^{\Psi}$. Hereinafter, a time interval $\Delta t=t-t_{0}$ shall be considered small if for all $j, \Delta t \ll \Delta x_{j}(t) / v_{j}$, where $\Delta x_{j}(t)=x_{j}^{\Psi}(t)-x_{j}\left(t_{0}\right)$ and $v_{j}=p_{j}\left(t_{0}\right) / m_{j}$. We are going to show that for short time intervals $\Delta t$ these solutions are, for given initial data $x_{0}, p_{0}$, identical to the solutions of the usual Hamilton equations

$$
\dot{x}=\nabla_{p} H(x, p, t) \quad, \quad \dot{p}=-\nabla_{x} H(x, p, t)
$$

up to the order $\mathcal{O}\left(\Delta t^{2}\right)$.

Because of the importance of this result we dignify it as a theorem: 
Theorem 1 Let $x^{\Psi}(t)=\left(x_{1}^{\Psi}(t), \ldots, x_{n}^{\Psi}(t)\right)$ and $p^{\Psi}(t)=\left(p_{1}^{\Psi}(t), \ldots, p_{n}^{\Psi}(t)\right)$ be the solution of Hamilton's equations for $H^{\Psi}=H+Q^{\Psi}$. For $t$ close to the initial time $t_{0}$ we have

$$
\begin{aligned}
& x_{j}^{\Psi}(t)=x_{0, j}+\frac{p_{0, j}}{m_{j}}\left(t-t_{0}\right)+\mathcal{O}\left(\left(t-t_{0}\right)^{2}\right) \\
& p_{j}^{\Psi}(t)=p_{0, j}-\frac{1}{m_{j}} \frac{\partial V}{\partial x_{j}}\left(x_{0}\right)\left(t-t_{0}\right)+\mathcal{O}\left(\left(t-t_{0}\right)^{2}\right) .
\end{aligned}
$$

Proof. We are going to prove these asymptotic formulas for $n=1$; the general case is a straightforward generalization, working separately on each coordinate. We notice that formula (47) is an immediate consequence of formula (48) since

$$
\dot{x}_{j}^{\Psi}=\frac{\partial H^{\Psi}}{\partial p_{j}}=\frac{p_{j}}{m_{j}} .
$$

To prove formula (48) we begin showing that we have

$$
\left.m \dot{x}^{\Psi}=m \frac{x^{\Psi}-x_{0}}{t-t_{0}}-\frac{1}{2} \frac{\partial V}{\partial x}\left(x_{0}\right)\left(t-t_{0}\right)+\mathcal{O}\left(\left(t-t_{0}\right)^{2}\right)\right) .
$$

Recalling that by the first formula (34) we have

$$
m \dot{x}^{\Psi}=\frac{\partial S}{\partial x}\left(x^{\Psi}, t\right)
$$

we replace $S$ with its approximation $\bar{S}$; taking (18) into account, this yields

$$
m \dot{x}^{\Psi}=m \frac{x^{\Psi}-x_{0}}{t-t_{0}}-\frac{\partial \bar{V}}{\partial x}\left(x^{\Psi}, x_{0}\right)\left(t-t_{0}\right)+\mathcal{O}\left(\left(t-t_{0}\right)^{2}\right) .
$$

By continuity we have $x^{\Psi}=x_{0}+\mathcal{O}\left(t-t_{0}\right)$, hence using formula (45),

$$
\begin{aligned}
\frac{\partial \bar{V}}{\partial x}\left(x^{\Psi}, x_{0}\right) & =\frac{\partial \bar{V}}{\partial x}\left(x_{0}, x_{0}\right)+\mathcal{O}\left(t-t_{0}\right) \\
& =\frac{1}{2} \frac{\partial V}{\partial x}\left(x_{0}\right)+\mathcal{O}\left(t-t_{0}\right)
\end{aligned}
$$

insertion of this value in (51) yields (49). We are now going to use equation (49) to derive formulas (47) and (48). Differentiating both sides of (49) with respect to time $t$ we get

$$
m \ddot{x}^{\Psi}=-m \frac{x^{\Psi}-x_{0}}{\left(t-t_{0}\right)^{2}}+m \frac{\dot{x}^{\Psi}}{t-t_{0}}-\frac{1}{2} \frac{\partial V}{\partial x}\left(x_{0}\right)+\mathcal{O}\left(t-t_{0}\right) ;
$$


replacing in this equality $\dot{x}^{\Psi}$ with the value given by (49) and simplifying leads to

$$
m \ddot{x}^{\Psi}=-\frac{\partial V}{\partial x}\left(x_{0}\right)+\mathcal{O}\left(t-t_{0}\right) .
$$

Integrating both sides from $t_{0}$ to $t$ yields

$$
m \dot{x}^{\Psi}-m \dot{x}^{\Psi}(0)=-\frac{\partial V}{\partial x}\left(x_{0}\right)\left(t-t_{0}\right)+\mathcal{O}\left(\left(t-t_{0}\right)^{2}\right)
$$

which is formula (48), since $p^{\Psi}=m \dot{x}^{\Psi}$.

The quantum potential is absent from the asymptotic solutions (47) and (48); this suggests that the latter is negligible for short times. Let us show that this is indeed the case. Recall that we are dealing with a pointlike source modeled by the Dirac delta centered at $x_{0}$; the wavefunction is thus the unique solution to Schrödinger's equation (2) with initial datum $\Psi\left(x, t_{0}\right)=\delta\left(x-x_{0}\right)$; in view of formula (9) expressing the solution in terms of the propagator $K$ we thus have

$$
\Psi(x, t)=\int K\left(x, x^{\prime}, t, t_{0}\right) \delta\left(x^{\prime}-x_{0}\right) d^{n} x^{\prime}=K\left(x, x_{0}, t, t_{0}\right)
$$

hence the wavefunction is the propagator $K\left(x, x_{0}, t, t_{0}\right)$ itself. Writing $\Psi=$ $\sqrt{\rho} e^{\frac{i}{\hbar} S}$ the wavefunction is thus

$$
\Psi(x, \Delta t)=\sqrt{\rho(x, \Delta t)} e^{\frac{i}{\hbar} S(x, \Delta t)}
$$

after time $\Delta t=t-t_{0}$. Taking into account the estimate (24) for the approximate short-time propagator (23)

$$
\bar{\Psi}(x, \Delta t)=\bar{K}\left(x, x_{0}, t, t_{0}\right)=\left(\frac{m_{1} \cdots m_{n}}{2 \pi i \hbar\left(t-t_{0}\right)}\right)^{n / 2} e^{\frac{i}{\hbar} \bar{S}\left(x, x_{0}, t, t_{t}\right)}
$$

we have, using the inequality ||$a|-| b|| \leq|a-b|$ (which is a straightforward consequence of the triangle inequality) with $a=|\Psi|, b=|\bar{\Psi}|$,

$$
|| \Psi(x, \Delta t)|-| \bar{\Psi}(x, \Delta t)|| \leq|\Psi(x, \Delta t)-\bar{\Psi}(x, \Delta t)|=\mathcal{O}\left(\Delta t^{2}\right)
$$

and hence

$$
\begin{aligned}
\sqrt{\rho(x, \Delta t)} & =\left(\frac{m_{1} \cdots m_{n}}{2 \pi i \hbar \Delta t}\right)^{n / 2}+\mathcal{O}\left(\Delta t^{2}\right) \\
& =\left(\frac{m_{1} \cdots m_{n}}{2 \pi i \hbar \Delta t}\right)^{n / 2}\left(1+\mathcal{O}\left(\Delta t^{\frac{n}{2}+2}\right)\right)
\end{aligned}
$$


It immediately follows that

$$
Q^{\Psi}(x, \Delta t)=\mathcal{O}\left(\Delta t^{\frac{n}{2}+2}\right)
$$

(the quantum potential $Q^{\Psi}(x, t)$ is not defined for $t=0$ when $x \neq x_{0}$ in the

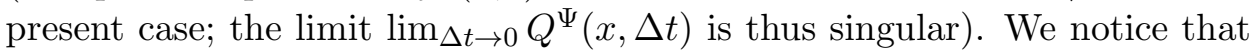
the quantum potential decreases for small times as the number of degrees of freedom increases; the effect of the quantum potential is thus negligible for systems consisting of a large number of particles and vanishes at the macroscopic scale. As will be further discussed in the last section, this might have further bearings on the way we understand decoherence.

That the quantum evolution becomes classical for infinitesimal times has been hinted by many authors; it was apparently known already to Feynman [18] (see the discussion in [64]); in [40] (p. 269) Holland has discussed this fact, by considering infinitesimal time intervals whose sequence constructs a finite path. He shows that along each segment the motion is classical due to a negligible quantum potential, and that it follows that the quantum path may be decomposed into a sequence of segments along each of which the classical action is a minimum.

In [55] Markowsky and Schopohl briefly discuss this in the context of cold Bose atoms around the crossing of quantum waveguides.

\subsection{The general case}

In the previous discussion we assumed that the wavefunction was perfectly localized at initial time: $\Psi\left(x, t_{0}\right)=\delta\left(x-x_{0}\right)$, in which case $\Psi(x, t)$ is just the propagator $K\left(x, x_{0}, t, t_{0}\right)$. Let us now consider the case where $\Psi\left(x, t_{0}\right)$ is an arbitrary wavepacket $\Psi_{0}(x)=\sqrt{\rho_{0}(x)} e^{i S_{0}(x) / \hbar}$. Writing the solution of Schrödinger's equation (2) as

$$
\Psi(x, t)=\sqrt{\rho(x, t)} e^{\frac{i}{\hbar} S(x, t)}
$$

the phase $S(x, t)$ satisfies the quantum Hamilton-Jacobi equation

$$
\frac{\partial S}{\partial t}(x, t)+\sum_{j=1}^{n} \frac{1}{2 m_{j}}\left(\frac{\partial S}{\partial x_{j}}(x, t)\right)^{2}+V(x)+Q^{\Psi}(x, t)=0
$$

where the quantum potential is

$$
Q^{\Psi}(x, t)=-\sum_{j=1}^{n} \frac{\hbar^{2}}{2 m_{j}} \frac{1}{\sqrt{\rho(x, t)}} \frac{\partial^{2} \sqrt{\rho(x, t)}}{\partial x_{j}^{2}} .
$$


The continuity equation is here

$$
\frac{\partial \rho(x, t)}{\partial t}+\sum_{j=1}^{n} \frac{1}{m_{j}} \frac{\partial}{\partial x_{j}}\left(\rho(x, t) \frac{\partial S(x, t)}{\partial x_{j}}\right)=0 .
$$

In terms of $\rho$, a time interval $\Delta t$ may be considered small if $\Delta t=t-t_{0} \ll$ $\rho(x, t) / \frac{\partial \rho(x, t)}{\partial t}$ for all $x$.

The Bohmian trajectory of the system of particles is determined by the equations

$$
m_{j} \dot{x}_{j}^{\Psi}=\frac{\partial S}{\partial x_{j}}\left(x^{\Psi}, t\right) \quad, x_{j}^{\Psi}\left(t_{0}\right)=x_{0, j} .
$$

One shows that again this motion is given in phase space by the quantum Hamilton equations

$$
\dot{x}_{j}^{\Psi}(t)=\frac{\partial H^{\Psi}}{\partial p_{j}}\left(x_{j}^{\Psi}(t), p_{j}^{\Psi}(t), t\right), \dot{p}_{j}^{\Psi}(t)=-\frac{\partial H^{\Psi}}{\partial x_{j}}\left(x_{j}^{\Psi}(t), p_{j}^{\Psi}(t), t\right)
$$

with initial conditions

$$
x_{j}^{\Psi}\left(t_{0}\right)=x_{0, j} \quad, \quad p_{j}^{\Psi}\left(t_{0}\right)=p_{0, j}=\frac{\partial S}{\partial x_{j}}\left(x_{0}, t_{0}\right) ;
$$

as before $H^{\Psi}=H+Q^{\Psi}$.

Let us now prove what can perhaps be seen as the main property of the flow determined by equations (56), namely that for short times the quantum potential is conserved along the trajectory up to an error term $\mathcal{O}\left(\left(t-t_{0}\right)^{2}\right)$. The analogue of Theorem 1 for the general case will follow.

Theorem 2 Let $\left(x_{0}, t_{0}\right)$ be a fixed initial point of space-time. The quantum potential $Q^{\Psi}$, calculated along the Bohmian trajectory $x^{\Psi}(t)$ satisfies

$$
Q^{\Psi}\left(x^{\Psi}(t), t\right)=Q^{\Psi}\left(x_{0}, t_{0}\right)+\mathcal{O}\left(\left(t-t_{0}\right)^{2}\right)
$$

for small $\Delta t=t-t_{0}$.

Proof. Writing $\rho^{1 / 2}(x, t)=\sqrt{\rho(x, t)}, x^{\Psi}(t)=x^{\Psi}$ the quantum potential at $\left(x_{0}, t_{0}\right)$ is

$$
Q^{\Psi}\left(x_{0}, t_{0}\right)=-\sum_{j=1}^{n} \frac{\hbar^{2}}{2 m_{j}} \frac{1}{\rho^{1 / 2}\left(x_{0}, t_{0}\right)} \frac{\partial^{2} \rho^{1 / 2}}{\partial x_{j}^{2}}\left(x_{0}, t_{0}\right)
$$


and at $\left(x^{\Psi}, t\right)$

$$
Q^{\Psi}\left(x^{\Psi}, t\right)=-\sum_{j=1}^{n} \frac{\hbar^{2}}{2 m_{j}} \frac{1}{\rho^{1 / 2}\left(x^{\Psi}, t\right)} \frac{\partial^{2} \rho^{1 / 2}}{\partial x_{j}^{2}}\left(x^{\Psi}, t\right) .
$$

Defining the velocity field $v(x, t)=\left(v_{1}(x, t), \ldots, v_{n}(x, t)\right)$ by

$$
m_{j} v_{j}(x, t)=\frac{\partial S(x, t)}{\partial x_{j}}
$$

the continuity equation (54) can be written in the usual form

$$
\frac{\partial \rho(x, t)}{\partial t}+\nabla_{x} \cdot(\rho(x, t) v(x, t))=0 .
$$

It follows that (Chorin and Marsden [11], §1.1)

$$
\rho\left(x^{\Psi}, t\right)=\rho\left(x_{0}, t_{0}\right)\left|\frac{d x^{\Psi}}{d x_{0}}\right|^{-1}
$$

where $d x^{\Psi} / d x_{0}$ is the determinant of the Jacobian matrix

$$
\frac{d x^{\Psi}}{d x_{0}}=\left(\frac{\partial x_{j}^{\Psi}}{\partial x_{0, k}}\right)_{1 \leq j, k \leq n}
$$

of the flow map $x_{0} \longmapsto x^{\Psi}$. Now,

$$
x_{j}^{\Psi}(t)=x_{0, j}+v_{0, j} \Delta t+\mathcal{O}\left(\Delta t^{2}\right)
$$

hence

$$
\frac{\partial x_{j}^{\Psi}(t)}{\partial x_{0, k}}=\delta_{j k}+\mathcal{O}\left(\Delta t^{2}\right)
$$

so that

$$
\left|\frac{d x^{\Psi}}{d x_{0}}\right|=\operatorname{det}\left(I+\mathcal{O}\left(\Delta t^{2}\right)\right)=1+\mathcal{O}\left(\Delta t^{2}\right)
$$

then (59) yields

$$
\rho\left(x^{\Psi}, t\right)=\rho\left(x_{0}, t_{0}\right)\left(1+\mathcal{O}\left(\Delta t^{2}\right)\right)
$$

and therefore also

$$
\rho^{1 / 2}\left(x^{\Psi}, t\right)=\rho^{1 / 2}\left(x_{0}, t_{0}\right)\left(1+\mathcal{O}\left(\Delta t^{2}\right)\right) .
$$


Differentiating this expression with respect to the initial variables $x_{0, j}$ we get, using the chain rule together with (60),

$$
\begin{aligned}
\frac{\partial}{\partial x_{0, j}}\left[\rho^{1 / 2}\left(x^{\Psi}, t\right)\right] & =\sum_{k=1}^{n} \frac{\partial \rho^{1 / 2}}{\partial x_{0, k}}\left(x^{\Psi}, t\right) \frac{\partial x_{k}^{\Psi}}{\partial x_{0, j}} \\
& =\frac{\partial \rho^{1 / 2}}{\partial x_{0, j}}\left(x^{\Psi}, t\right)\left(1+\mathcal{O}\left(\Delta t^{2}\right)\right)
\end{aligned}
$$

repeating the same argument with $\rho^{1 / 2}$ replaced with $\partial \rho^{1 / 2} / \partial x_{0, j}$ we get

$$
\frac{\partial^{2}}{\partial x_{0, j}^{2}}\left[\rho^{1 / 2}\left(x^{\Psi}, t\right)\right]=\frac{\partial^{2} \rho^{1 / 2}}{\partial x_{0, j}^{2}}\left(x^{\Psi}, t\right)\left(1+\mathcal{O}\left(\Delta t^{2}\right)\right)
$$

and hence

$$
\frac{\partial^{2} \rho^{1 / 2}}{\partial x_{0, j}^{2}}\left(x^{\Psi}, t\right)=\frac{\partial^{2}}{\partial x_{0, j}^{2}}\left[\rho^{1 / 2}\left(x^{\Psi}, t\right)\right]\left(1+\mathcal{O}\left(\Delta t^{2}\right)\right) ;
$$

in view of (62) we thus have

$$
\frac{\partial^{2} \rho^{1 / 2}}{\partial x_{0, j}^{2}}\left(x^{\Psi}, t\right)=\frac{\partial^{2} \rho^{1 / 2}}{\partial x_{0, j}^{2}}\left(x_{0}, t_{0}\right)\left(1+\mathcal{O}\left(\Delta t^{2}\right)\right) .
$$

Formula (57) follows.

A practical consequence of this result, might be the short time simulation of various external potentials by shaping the initial wave packet to create the suitable quantum potential. If one prepares the initial wavefunction with a shape corresponding to a specific quantum potential, then according to the above theorem, this potential might remain a good approximation for the actual one along some short time interval.

We note that the crucial step in the proof above is formula (61), which follows from property (59) of the solution of the continuity equation; all the other estimates we needed followed from it. Here is another independent way to prove (61); we limit ourselves to the case $n=1$, in which we have

$$
\begin{aligned}
\frac{d}{d t} \rho\left(x^{\Psi}, t\right) & =\frac{\partial \rho}{\partial t}\left(x^{\Psi}, t\right)+\frac{\partial \rho}{\partial x}\left(x^{\Psi}, t\right) \dot{x}^{\Psi} \\
& =-\frac{\partial}{\partial x}\left(\rho\left(x^{\Psi}, t\right) \dot{x}^{\Psi}\right)+\frac{\partial \rho}{\partial x}\left(x^{\Psi}, t\right) \dot{x}^{\Psi} \\
& =\rho\left(x^{\Psi}, t\right) \frac{\partial \dot{x}^{\Psi}}{\partial x} .
\end{aligned}
$$


We have $x^{\Psi}=x_{0}+v_{0} t+\mathcal{O}\left(\Delta t^{2}\right)$, hence $\dot{x}^{\Psi}=v_{0}+\mathcal{O}(\Delta t)$ and $\partial \dot{x}^{\Psi} / \partial x=$ $\mathcal{O}(\Delta t)$, from which follows that

$$
\frac{d}{d t} \rho\left(x^{\Psi}, t\right)=\mathcal{O}(\Delta t)
$$

formula (61) follows.

As observed in [33] and 34], this suggests for short times the incompressibility of the quantum fluid, which implies non-spreading of the wavepacket and a constant Shannon entropy.

We now prove the following generalization of Theorem 1 for the short time solutions of (56):

Theorem 3 Let $x^{\Psi}(t)=\left(x_{1}^{\Psi}(t), \ldots, x_{n}^{\Psi}(t)\right)$ and $p^{\Psi}(t)=\left(p_{1}^{\Psi}(t), \ldots, p_{n}^{\Psi}(t)\right)$ be the solution at time $t$ of Hamilton's equations for $H^{\Psi}=H+Q^{\Psi}$ where $Q^{\Psi}$ is given by (53). (i) We have

$$
\begin{aligned}
& x_{j}^{\Psi}(t)=x_{0, j}+\frac{p_{0, j}}{m_{j}} \Delta t+\mathcal{O}\left(\Delta t^{2}\right) \\
& p_{j}^{\Psi}(t)=p_{0, j}-\frac{\partial V}{\partial x_{j}}\left(x_{0}\right) \Delta t+\mathcal{O}\left(\Delta t^{2}\right) ;
\end{aligned}
$$

(ii) The quantum flow $\left(f_{t, t_{0}}^{\Psi}\right)$ is approximated to order $\mathcal{O}\left(\Delta t^{2}\right)$ by the usual Hamiltonian flow $\left(f_{t, t_{0}}\right)$ :

$$
f_{t, t_{0}}^{\Psi}\left(x_{0}, p_{0}\right)=f_{t, t_{0}}\left(x_{0}, p_{0}\right)+\mathcal{O}\left(\Delta t^{2}\right)
$$

Proof. We again limit ourselves to the case $n=1$. As in the proof of Theorem 1, formula (63) immediately follows from formula (64). The latter is a consequence of (57), since we then have, using the explicit expression of $H^{\Psi}$

$$
\begin{aligned}
\dot{p}^{\Psi}(t) & =-\frac{\partial V}{\partial x}\left(x^{\Psi}(t)\right)-\frac{\partial Q}{\partial x}\left(x^{\Psi}, t\right) \\
& =-\frac{\partial V}{\partial x}\left(x^{\Psi}(t)\right)-\frac{\partial}{\partial x} Q\left(x_{0}, t_{0}\right)+\mathcal{O}\left(\Delta t^{2}\right) \\
& =-\frac{\partial V}{\partial x}\left(x^{\Psi}(t)\right)+\mathcal{O}\left(\Delta t^{2}\right)
\end{aligned}
$$

because $Q\left(x_{0}, t_{0}\right)$ is constant. Formula (65) follows since the phase space transformations $g_{t, t_{0}}$ defined by $g_{t, t_{0}}\left(x_{0}, p_{0}\right)=\left(x\left(t, t_{0}\right), p\left(t, t_{0}\right)\right)$ with

$$
x_{j}\left(t, t_{0}\right)=x_{0, j}+\frac{p_{0, j}}{m_{j}} \Delta t, p_{j}\left(t, t_{0}\right)=p_{0, j}-\frac{\partial V}{\partial x_{j}}\left(x_{0}\right) \Delta t
$$

are themselves an $\mathcal{O}\left(\Delta t^{2}\right)$ approximation to the time-dependent Hamiltonian flow $\left(f_{t, t_{0}}\right)$ in view of Taylor's theorem. 


\section{Quantum Zeno Effect}

The quantum Zeno effect is widely discussed in the literature [6, 43, 44, 47]. It has been claimed by Misra and Sudarshan [57, 9] that the quantum Zeno effect leads to the conclusion that, and we quote, "an unstable particle observed continuously whether it has decayed or not will never be found to decay". These authors confine their discussion specifically to the case of $\alpha$ decay in a cloud or bubble chamber. Peres [61] has considered a more general question, namely, "If an unstable quantum system is kept under continuous observation, will it decay?" We will see how the question raised by Misra and Sudarshan [57] in their discussion on $\alpha$-decay can be answered using the Bohm-Hiley approach [8]. In order to inhibit the occurrence of $\alpha$-decay, we must use a process that directly interacts with the unstable nuclei. It is not sufficient to surround the nuclei with a passive detection device like a bubble or cloud chamber. Merely detecting the $\alpha$-particle outside the nucleus is not sufficient to inhibit the decay.

In de Gosson and Hiley [26, 28] it was assumed that the initial wavepacket $\Psi_{0}$ was a point source modelled by the propagator itself; here we consider the more realistic situation where $\Psi_{0}$ is an arbitrary wavepacket. For this we will apply the machinery developed in Section 3.4 together with a generalization of the Lie-Trotter formula.

\subsection{The Lie-Trotter formula for time-dependent flows}

We are going to prove a rather straightforward extension of the usual LieTrotter [66] formula for flows to the time-dependent case; we will use this result in the next section as we deal with the quantum Zeno effect. Recall (Abraham et al. [2, Chorin et al. [10] and also Appendix B in de Gosson [21]) that given a vector field $X=X(x, p)=(a(x, p), b(x, p))$ on an open subset $\Omega$ of $\mathbb{R}_{x, p}^{2 n}$ (or any other Euclidean space, for that matter) a family $\left(g_{t}\right)$ of functions $\Omega \longrightarrow \mathbb{R}^{2 n}$ is called an algorithm for the flow $\left(f_{t}\right)$ determined by the vector field $X$ if for every point $z_{0}=\left(x_{0}, p_{0}\right)$ in $\Omega$ we have

$$
X\left(z_{0}\right)=\left.\frac{\partial}{\partial t} k_{t}\left(z_{0}\right)\right|_{t=0} .
$$

In this case the sequence of iterates $\left(k_{t / N}\left(z_{0}\right)\right)^{N}$ converges towards $f_{t}\left(z_{0}\right)$ :

$$
f_{t}\left(z_{0}\right)=\lim _{N \rightarrow \infty}\left(k_{t / N}\left(z_{0}\right)\right)^{N} .
$$

Applying this result to the case where the vector field $X$ is generated by a time-independent Hamiltonian $H$, that is $X=\left(\nabla_{p} H,-\nabla_{x} H\right)$, formula 
(67) yields a convenient procedure for approximating Hamiltonian flows. In our context the trouble is that we would like to apply the same kind of procedure to the Hamiltonian function $H^{\Psi}=H+Q^{\Psi}$, which is timedependent because of the presence of the quantum potential $Q^{\Psi}$; as we have seen, the flow determined by such a Hamiltonian function is no longer a one-parameter group (this is due to the fact that the Hamiltonian vector field is here time-dependent: $X_{t}=X(x, p, t)$ and thus it is not a vector field in the usual sense. The way out of this difficulty consists of lifting the flow to the time-dependent phase space $\mathbb{R}_{x, p, t}^{2 n+1}$ : defining the "suspended vector field" $\widetilde{X}=(X, 1)$ (Abraham and Marsden [1]) the flow $\left(\widetilde{f}_{t}\right)$ it determines is given by the formula

$$
\widetilde{f}_{t}\left(z_{0}, t_{0}\right)=\left(f_{t+t_{0}, t_{0}}\left(z_{0}\right), t+t_{0}\right)
$$

where, as usual $f_{t, t^{\prime}}=f_{t}\left(f_{t^{\prime}}\right)^{-1}$ is the time-dependent flow of $X_{t}$. On calls $\left(\widetilde{f}_{t}\right)$ the suspended flow of $X_{t}$. Now,

$$
\begin{aligned}
\tilde{f}_{t} \tilde{f}_{t^{\prime}}\left(x_{0}, p_{0}, t_{0}\right) & =\widetilde{f}_{t}\left(f_{t^{\prime}+t_{0}, t_{0}}\left(z_{0}\right), t^{\prime}+t_{0}\right) \\
& =\left(f_{t+t^{\prime}+t_{0}, t^{\prime}+t_{0}} f_{t^{\prime}+t_{0}, t_{0}}\left(z_{0}\right), t+t^{\prime}+t_{0}\right) \\
& =\left(f_{t+t^{\prime}+t_{0}, t_{0}}\left(z_{0}\right), t+t^{\prime}+t_{0}\right) \\
& =\widetilde{f}_{t+t^{\prime}}\left(z_{0}, t_{0}\right)
\end{aligned}
$$

hence $\left(\widetilde{f_{t}}\right)$ is a one-parameter group, that is a flow in the usual sense, to which we may apply the Lie-Trotter formula.

Theorem 4 Let $X_{t}=(a(x, p, t), b(x, p, t))$ be a time dependent vector field on phase space $\mathbb{R}_{x, p}^{2 n}$ and $\left(f_{t, t_{0}}\right)$ its time-dependent flow. Let $\left(k_{t, t_{0}}\right)$ be a two-parameter family of transformations $\mathbb{R}_{x, p}^{2 n} \longrightarrow \mathbb{R}_{x, p}^{2 n}$. If

$$
X_{t_{0}}\left(z_{0}\right)=\left.\frac{\partial}{\partial t} k_{t, t_{0}}\left(z_{0}\right)\right|_{t=t_{0}}
$$

then we have

$$
\lim _{N \rightarrow \infty}\left(k_{t+t_{0}, t_{N-1}+t_{0}} k_{t_{N-1}+t_{0}, t_{N-2}+t_{0}} \cdots k_{t_{1}, t_{0}}\left(z_{0}\right)\right)=f_{t, t_{0}}\left(z_{0}\right)
$$

for every $z_{0}=\left(x_{0}, p_{0}\right)$, where $t_{1}, t_{2}, \ldots, t_{N-1}$ is a subdivision of the interval $\left[t_{0}, t\right]$ such that $\left|t_{j+1}-t_{j}\right|=\left|t-t_{0}\right| / N$.

Proof. Let $\widetilde{k}_{t}: \mathbb{R}_{x, p, t}^{2 n+1} \longrightarrow \mathbb{R}_{x, p, t}^{2 n+1}$ be defined by

$$
\widetilde{k}_{t}\left(z_{0}, t_{0}\right)=\left(k_{t+t_{0}, t_{0}}\left(z_{0}\right), t+t_{0}\right) ;
$$


we have

$$
\begin{aligned}
\left.\frac{\partial}{\partial t} \widetilde{k}_{t}\left(z_{0}, t_{0}\right)\right|_{t=t_{0}} & =\left(\left.\frac{\partial}{\partial t} k_{t+t_{0}, t_{0}}\left(z_{0}\right)\right|_{t=t_{0}}, t_{0}\right) \\
& =\left(X_{t_{0}}\left(z_{0}\right), t_{0}\right) \\
& =\widetilde{X}\left(z_{0}, t_{0}\right)
\end{aligned}
$$

hence $\left(\widetilde{k}_{t}\right)$ is an algorithm for the suspended flow $\left(\widetilde{f}_{t}\right)$ of $X_{t}$. Applying the conventional Lie-Trotter formula (67) to the algorithm $\left(\widetilde{k}_{t}\right)$ we have

$$
\widetilde{f}_{t}\left(z_{0}, t_{0}\right)=\lim _{N \rightarrow \infty}\left(\widetilde{k}_{t / N}\right)^{N}\left(z_{0}, t_{0}\right) ;
$$

Using formulas (71) and (68) one easily shows by induction on the integer $N$ that

$$
\begin{aligned}
\left(\widetilde{k}_{t / N}\right)^{N}\left(z_{0}, t_{0}\right) & =\left(k_{t+t_{0}, \frac{(N-1) t}{N}+t_{0}} k_{\frac{(N-1) t}{N}+t_{0}, \frac{(N-2) t}{N}+t_{0}} \cdots k_{\frac{t}{N}+t_{0}, t_{0}}\left(z_{0}\right), t+t_{0}\right) \\
& =k_{t+t_{0}, t_{N-1}+t_{0}} k_{t_{N-1}+t_{0}, t_{N-2}+t_{0}} \cdots k_{t_{1}, t_{0}}\left(z_{0}\right)
\end{aligned}
$$

hence

$$
\left(f_{t+t_{0}, t_{0}}\left(z_{0}\right), t+t_{0}\right)=\lim _{N \rightarrow \infty}\left(k_{t, t_{N-1}} \cdots k_{t_{2}, t_{1}} k_{t_{1}, t_{0}}\left(z_{0}\right)\right)
$$

which proves the generalized Lie-Trotter formula (70).

\subsection{The main result}

We now choose an initial time $t_{0}$ and denote the corresponding quantum potential by $Q^{0}$ :

$$
Q^{0}(x, t)=-\sum_{j=1}^{n} \frac{\hbar^{2}}{2 m_{j}} \frac{1}{\sqrt{\rho\left(x, t_{0}\right)}} \frac{\partial^{2} \sqrt{\rho\left(x, t_{0}\right)}}{\partial x_{j}^{2}} .
$$

We set $H^{0}=H+Q^{0}$. After time $\Delta t=\left(t-t_{0}\right) / N$ the particle is observed at a point $x_{1}$, and the new wavefunction is now $\Psi_{1}=\sqrt{\rho_{1}} e^{i S_{1} / \hbar}$ hence the future quantum evolution of this particle will be governed by the new Hamiltonian $H^{1}=H+Q^{1}$, where

$$
Q^{1}(x, t)=-\sum_{j=1}^{n} \frac{\hbar^{2}}{2 m_{j}} \frac{1}{\sqrt{\rho_{1}(x, t)}} \frac{\partial^{2} \sqrt{\rho_{1}(x, t)}}{\partial x_{j}^{2}}
$$

together with the guiding condition $p_{1}=\nabla_{x} S_{1}$. Repeating this procedure until time $t$ we obtain a sequence of points $x_{0}, x_{1}, x_{2}, \ldots, x_{N}=x$ corresponding to the successive observations at times $t_{0}, t_{1}=t_{0}+\Delta t, t_{2}=t_{0}+2 \Delta t, \ldots$, 
t. Let $H^{0}, H^{1}, \ldots, H^{N}$ be the sequence of Hamiltonian functions determined by the quantum potentials $Q^{0}, Q^{1}, \ldots, Q^{N-1}$ :

$$
H^{0}=H+Q^{0}, H^{1}=H+Q^{1}, \ldots, H^{N-1}=H+Q^{N-1} ;
$$

we denote by $\left(f_{t, t_{0}}^{0}\right),\left(f_{t, t_{1}}^{1}\right), \ldots,\left(f_{t, t_{N-1}}^{N-1}\right)$ the corresponding time-dependent flows. Writing $z_{0}=\left(x_{0}, p_{0}\right), z_{1}=\left(x_{1}, p_{1}\right)$, etc. we thus have a sequence of successive equalities

$$
z_{1}=f_{t_{1}, t_{0}}^{0}\left(z_{0}\right), z_{2}=f_{t_{2}, t_{1}}^{1}\left(z_{1}\right), \ldots, z_{N}=f_{t, t_{N-1}}^{N-1}\left(z_{N-1}\right)
$$

where $p_{1}=\nabla_{x} S_{1}, p_{2}=\nabla_{x} S_{2}, \ldots, p_{N-1}=\nabla_{x} S_{N-1}$, which implies that the final position $x=x_{N}$ of the particle at time $t$ is expressed in terms of the initial point $x_{0}$ by the formula

$$
(x, p)=f_{t, t_{N-1}}^{N-1} \cdots f_{t_{2}, t_{1}}^{1} f_{t_{1}, t_{0}}^{0}\left(x_{0}, p_{0}\right)
$$

(notice that the intermediate points $t_{1}, t_{2}, \ldots, t_{N-1}$ depend on $t$ ).

Theorem 5 Let the phase space transformations $f_{t_{1}, t_{0}}^{0}, f_{t_{2}, t_{1} \ldots,}^{1} f_{t, t_{N-1}}^{N-1}$ be defined as above, $t_{1}, t_{2}, \ldots, t_{N-1}$ being a subdivision of the interval $\left[t_{0}, t\right]$ such that $\Delta t=t_{j+1}-t_{j}=\left(t-t_{0}\right) / N$. The two-parameter family of transformations $k_{t, t_{0}}$ defined by

$$
k_{t, t_{0}}\left(z_{0}\right)=f_{t, t_{N-1}}^{N-1} \cdots f_{t_{2}, t_{1}}^{1} f_{t_{1}, t_{0}}^{0}\left(z_{0}\right)
$$

is an algorithm for the flow $\left(f_{t}\right)$ determined by the classical Hamiltonian $H$.

Proof. It is sufficient to show that for small $\Delta t=t-t_{0}$ we have

$$
k_{t, t_{0}}\left(z_{0}\right)=f_{t, t_{0}}\left(z_{0}\right)+\mathcal{O}\left(\Delta t^{2}\right)
$$

for then

$$
\begin{aligned}
\left.\frac{\partial}{\partial t} k_{t, t_{0}}\left(z_{0}\right)\right|_{t=t_{0}} & =\lim _{\Delta t \rightarrow 0} \frac{k_{t_{0}+\Delta t, t_{0}}\left(z_{0}\right)-z_{0}}{\Delta t} \\
& =\lim _{\Delta t \rightarrow 0} \frac{f_{t_{0}+\Delta t, t_{0}}\left(z_{0}\right)-z_{0}}{\Delta t} \\
& =X_{t}\left(z_{0}\right)
\end{aligned}
$$

and $\left(k_{t, t_{0}}\right)$ is then indeed an algorithm for $\left(f_{t, t_{0}}\right)$ in view of Theorem 4. In light of Theorem 3 (formula (65)), each transformation $f_{t_{j+1}, t_{j}}^{j}(z)$ is approximated to order $\mathcal{O}\left(\left(t_{j+1}-t_{j}\right)^{2}\right)=\frac{1}{N} \mathcal{O}\left(\Delta t^{2}\right)$ by $f_{t_{j+1}, t_{j}}(z)$, hence

$$
\begin{aligned}
k_{t, t_{0}}\left(z_{0}\right) & =f_{t, t_{N-1}}^{N-1} \cdots f_{t_{2}, t_{1}}^{1} f_{t_{1}, t_{0}}^{0}\left(z_{0}\right) \\
& =f_{t, t_{N-1}} \cdots f_{t_{2}, t_{1}} f_{t_{1}, t_{0}}\left(z_{0}\right)+\mathcal{O}\left(\Delta t^{2}\right)
\end{aligned}
$$


which proves the estimate (74).

We have shown that

$$
\lim _{N \rightarrow \infty} f_{t, t_{N-1}}^{N-1} \cdots f_{t_{2}, t_{1}}^{1} f_{t_{1}, 0}^{0}\left(x_{0}, p_{0}\right)=f_{t}\left(x_{0}, p_{0}\right)
$$

the physical interpretation is that if one performs a series of observations of the particle at very short time intervals, then the recorded trajectory is the classical one. This is in agreement with the usual interpretation of the quantum Zeno effect ("the watched pot never boils"): an almost continuous observation of a particle (or system of particles) precludes the development of a quantum trajectory.

\subsection{The Mott problem}

The Mott problem (or paradox) illustrates in a striking manner the difficulties of understanding the nature of wavepacket measurement. The story goes back to 1929, when Heisenberg and Mott [59, 35] reflected on the following problem: in a Wilson cloud chamber, $\alpha$-particles are emitted from nuclei of radioactive atoms; due to radial symmetry of the problem, the $\alpha$ particles are represented by spherical wavefunctions. These particles create droplets of condensation when interacting with water molecules in the vapor, thus creating a track of condensation (see also Dell'Antonio [12]). Since the spherical wavefunction spreads isotropically in all directions it could have been expected to randomly ionize the water molecules, leaving behind a spherical track. However, when one actually performs the experiment one always finds a single linear track. In [4, Bell illustrates the situation by comparing the cloud chamber with a stack of photographic plates: to produce this straight trajectory, the ionized gas molecules are assumed to act as an array of potential measuring devices, leaving a record of the track of the $\alpha$-particle on the stack of plates. We can thus regard the $\alpha$-particle as being "continuously watched". This means, in a sense, that continuous observation "dequantizes" quantum trajectories. The frequent observations render the dynamic contribution of the quantum potential negligible. This property is, of course, essentially a consequence of the quantum Zeno effect, which has been shown to inhibit the decay of unstable quantum systems when under continuous observation (see [8, 17, 30, 32]). Another illustration of how continuous observations of a different kind can give rise to a quantum Zeno effect has already been given in Bohm and Hiley [8]. They considered the transition of an Auger-like particle and showed that the perturbed wavefunction, which is proportional to $\Delta t$ for times less that $1 / \Delta E,(\Delta E$ is 
the energy released in the transition) will never become large and therefore cannot make a significant contribution to the quantum potential necessary for the transition to occur. Thus again, the reason that no transition will take place is the vanishing of the quantum potential.

\section{Discussion}

We have shown that the quantum potential plays a key role in determining the behaviour of a quantum particle. This potential is not a mere "add-on" but an essential feature that is a necessary consequence of the relationship between the symplectic group structure (classical mechanics) and its double cover, the metaplectic group and its non-linear generalisation. Specifically there is a deep relation between the Hamilton flows, $f_{t t^{\prime}}$, of classical physics and the flows $f_{t t^{\prime}}^{\psi}$ associated with the quantum behaviour in the covering space [23, 27]. It is this feature that is key to understanding the relationship between a particle that exhibits only classical behaviour and one that gives rise to quantum phenomena.

As is well known at the particle level, we have lost causality in quantum phenomena but it has been replaced by what we will call "wave causality", which is defined through the Schrödinger equation. However the real part of this equation under polar decomposition appears to have restored causality at the particle level. How can this be?

The answer lies in the two observations we make in section 3.2 about the relationship between the two types of flow, $f_{t, t^{\prime}}$ and $f_{t t^{\prime}}^{\psi}$. This approach gives rise to a very different way of analysing quantum phenomena from the conventional approaches, including the approach of Bohmian mechanics as detailed in Dürr and Teufel [16]. Our two observations referred to the extensive work of one of us (MdG [21]), who has shown in very general terms that causality as defined by Chapman-Kolmogorov is retained in the covering space by the appearance of an additional term, $Q^{\psi}$. Note that the Schrödinger equation operates in this covering space [29]. Thus it is a necessary feature in the relationship between classical and quantum mechanics. Having established this relationship, it is now clear that once $Q^{\psi}$ becomes small in relation to the kinetic energy, the phenomena loses it quantum signature and becomes classical.

In this context we have analysed the short time behaviour of the quantum potential and shown that the suppression of $Q^{\psi}$ is possible if the successive positions of a particle can be defined in a short enough time. To see this, 
we must examine equations (63)-(64), that is

$$
\begin{aligned}
& x_{j}^{\Psi}(t)=x_{0, j}+\frac{p_{0, j}}{m_{j}} \Delta t+\mathcal{O}\left(\Delta t^{2}\right) \\
& p_{j}^{\Psi}(t)=p_{0, j}-\frac{\partial V}{\partial x_{j}}\left(x_{0}\right) \Delta t+\mathcal{O}\left(\Delta t^{2}\right) .
\end{aligned}
$$

Notice that there is no quantum potential present in either equation. Only when we allow higher order terms does the quantum potential appear. Thus if it is possible to obtain information of succession of positions in a short enough time without deflecting the particle significantly, then equation (70) shows that no quantum potential will appear and the trajectory will be a classical trajectory. In other words the quantum Zeno effect arises because $Q^{\Psi}$ is prevented from contributing to the process.

The short time behaviour we have discussed in this paper should not be confused with a discussion of the dominance of classical mechanics in the world we see around us. Classical phenomena can arise in spite of the presence of a quantum potential energy, provided the kinetic energy is much larger, in which case the effect of this potential can be neglected. This can be seen by examining equation (11) which clearly reduces to the classical Hamilton-Jacobi equation if the quantum potential energy term is small enough.

The magnitude of the quantum potential energy also accounts for the fragility of entangled states, where the non-local behaviour of these states is completely accounted for by the quantum potential energy. The exception to this fragility arises when an entangled state produces a stable state such as occurs in, for example, the electron pair in the ground state of a helium atom. In this case, a significant amount of energy is needed to overcome the quantum potential energy. This is in contrast to an EPR entangled pair of photons where great care must be taken to protect the entangled pairs from environmental effects. (See for example, Xiao-Song et al. [69] )

\section{Acknowledgments.}

Maurice de Gosson has been supported by a research grant from the Austrian Research Agency FWF (Projektnummer P27773-N13). Basil J. Hiley would like to thank the Fetzer Franklin Fund of the John E. Fetzer Memorial Trust for their support. Eliahu Cohen was supported by ERC AdG NLST. 


\section{References}

[1] R. Abraham, J. E. Marsden, Foundations of mechanics, Reading, Massachusetts: Benjamin/Cummings Publishing Company, 1978.

[2] R. Abraham, J. E. Marsden, T. Ratiu, Manifolds, Tensor Analysis, and Applications, Applied Mathematical Sciences 75 Springer, 1988.

[3] V. I. Arnold, Mathematical Methods of Classical Mechanics. Graduate Texts in Mathematics, second edition, Springer-Verlag, 1989.

[4] J. Bell, Speakable and unspeakable in quantum mechanics: Collected papers on quantum philosophy. Cambridge University Press, 2004.

[5] K. Y. Bliokh, A. Y. Bekshaev, A. G. Kofman, and F. Nori, Photon trajectories, anomalous velocities and weak measurements: a classical interpretation, New J. Physics, 15 (2013) 073022.

[6] E. Block and P. R. Berman, Quantum Zeno effect and quantum Zeno paradox in atomic physics, Phys. Rev. A 44 (1991) 1466-72.

[7] D. Bohm, B. J. Hiley and P. N. Kaloyerou, An Ontological Basis for the Quantum Theory: II. A Causal Interpretation of Quantum Fields, Phys. Reports, 144, (1987) 349-375.

[8] D. Bohm and B. Hiley, The Undivided Universe: An Ontological Interpretation of Quantum Theory. London \& New York: Routledge (1993).

[9] C. B. Chiu, E. C. G. Sudarshan, and B. Misra, Time evolution of unstable quantum states and a resolution of Zeno's paradox, Phys. Rev. D 16, (1977) 520-29.

[10] A. J. Chorin, T. J. R. Hughes, M. F. McCracken, and J. E. Marsden, Product formulas and numerical algorithms. Comm. Pure and Appl. Math. 31(2) (1978), 205-56.

[11] A. J. Chorin and J. E. Marsden, A mathematical introduction to fluid mechanics. Vol. 3. New York: Springer, 1990.

[12] G. F. Dell'Antonio, On Tracks in a Cloud Chamber, Found. Phys. 45 (2015), 11-21.

[13] Dirac, P. A. M., On the analogy between Classical and Quantum Mechanics, Rev. Mod. Phys., 17 (1945) 195-199. 
[14] Shin-ichi Doi, On the Cauchy problem for Schrödinger type equations and the regularity of solutions, J. Math. Kyoto Univ. (JMKYAZ) 34(2) (1994) 319-328.

[15] I. M. Duck, P. M. Stevenson and E. C. G. Sudarshan, The sense in which a "weak measurement" of a spin-1/2 particle's spin component yields a value 100, Phys. Rev., 40 (1989) 2112-2117.

[16] D.Dürr and S. Teufel, Bohmian mechanics. Springer Berlin Heidelberg, 2009.

[17] P. Facchi and S. Pascazio, Quantum Zeno dynamics: mathematical and physical aspects. J. Phys. A 41 (2008), 493001-005.

[18] R. P. Feynman and A. R. Hibbs, Quantum mechanics and path integrals, McGraw-Hill, New York, 1965.

[19] C. Garrod, Hamiltonian Path-Integral Methods, Rev. Mod. Phys. 38(3) (1966) 483-494.

[20] H. Goldstein, Classical Mechanics. Addison-Wesley, 1950, 2nd edition, (1980), 3d edition, 2002.

[21] M. de Gosson, The Principles of Newtonian and quantum mechanics. The need for Planck's constant $\hbar$. With a foreword by Basil Hiley. Imperial College Press, London, 2001.

[22] M. de Gosson, Symplectic Geometry and Quantum Mechanics, Birkhäuser, Basel, series "Operator Theory: Advances and Applications" (subseries: "Advances in Partial Differential Equations"), Vol. 166, (2006).

[23] M. de Gosson, Paths of Canonical Transformations and their Quantization. Rev. Math. Phys. 27(6), 1530003 (2015).

[24] M. de Gosson, Introduction to Born-Jordan Quantization, SpringerVerlag, series Fundamental Theories of Physics, February 2016.

[25] M. de Gosson, From Weyl to Born-Jordan quantization: The Schrödinger representation revisited, Phys. Reps. (2016).

[26] M. de Gosson and B. J. Hiley. Short-time quantum propagator and Bohmian trajectories. Phys. Lett. A 377(42) (2013) 3005-08. 
[27] M. de Gosson and B. J. Hiley, Imprints of the Classical World in Classical Mechanics, Foundations of Physics, 41, (2011), 1415-1436. arXiv:quant-ph/1001.4632.

[28] M. de Gosson, B. J. Hiley. Hamiltonian flows, short-time quantum propagators and the quantum Zeno effect. EmQM13: Emergent Quantum Mechanics 2013, J. Phys. Conf. Ser. 504 (2013) 012027.

[29] V. W. Guillemin and S. Sternberg, Symplectic Techniques in Physics, Cambridge University Press, Cambridge (1984)

[30] K. Gustafson, A Zeno story. arXiv:quant-ph/0203032.

[31] M. C. Gutzwiller, Chaos in classical and quantum mechanics. Springer Science \& Business Media, 2013.

[32] K. C. Hannabuss, An introduction to quantum theory. Oxford graduate texts in mathematics; 1. Oxford, 1997.

[33] E. Heifetz and E. Cohen, Toward a Thermo-hydrodynamic Like Description of Schrödinger Equation via the Madelung Formulation and Fisher Information, Foundations of Physics, Volume 45(11), 1514-25 (2015).

[34] E. Heifetz, R. Tsekov, E. Cohen, Z. Nussinov, On entropy production in the Madelung fluid and the role of Bohm's potential in classical diffusion, Preprint arXiv:1509.01265, 2015; Found. Phys. (2016) [in print].

[35] W. Heisenberg, Physical Principles of the Quantum Theory, Chicago 1930 [Courier Corporation, 2013].

[36] B. J. Hiley, Non-Commutative Geometry, the Bohm Interpretation and the Mind-Mater Relationship, in Proc. CASYS'2000, Liège, Belgium, Aug. 7-12, 2000.

[37] B. J. Hiley and R. E. Callaghan, Delayed-choice experiments and the Bohm approach. Phys. Scr. 74 (2006), 336-48.

[38] B. J. Hiley, R. E. Callaghan, and O. J. E. Maroney, Quantum trajectories, real, surreal or an approximation to a deeper process? quant-ph/0010020.

[39] B. J. Hiley, Weak Values:Approach through the Clifford and Moyal Algebras, J. Phys.: Conference Series, 361 (2012) 012014. 
[40] P. R. Holland, The quantum theory of motion. An account of the de Broglie-Bohm causal interpretation of quantum mechanics. Cambridge University Press, Cambridge, 1995.

[41] P. R. Holland, Hamiltonian theory of wave and particle in quantum mechanics I: Liouville's theorem and the interpretation of the de BroglieBohm theory. Nuovo Cimento B 116, 1043-70 (2001).

[42] P. R. Holland, Hamiltonian theory of wave and particle in quantum mechanics II: Hamilton-Jacobi theory and particle back-reaction. Nuovo Cimento B 116, 1143-1172 (2001).

[43] W. M. Itano, D. J. Heinzen, J. J. Bollinger, and D. J. Wineland, Quantum Zeno Effect, Phys. Rev. A 41, (1990) 2295-2300.

[44] E. Joos, Continuous measurement: Watchdog Effect and the Golden Rule, Phys. Rev. D 29, (1984) 1626-33.

[45] P. N. Kaloyerou, (1994) The Causal Interpretation of the Electromagnetic Field, Phys. Rep. 244, 287-385.

[46] T. Kato, Fundamental properties of Hamiltonian operators of Schrödinger type. Trans. Amer. Math. Soc. 70(2) (1951) 195-211.

[47] K. Kraus, Measuring Processes in Quantum Mechanics 1. Continuous Observation and the Watchdog Effect, Found. Phys. 11, (1981) 547-76.

[48] N. Kemmer, The particle aspects of meson theory, Proc. Roy. Soc., A 173 (1939) 91-116.

[49] E. H. Kerner and W. G. Sutcliffe, Unique Hamiltonian Operators via Feynman Path Integrals, J. Math. Phys. 11, (1970) 391-393.

[50] S. Kocsis, B. Braverman, S. Ravets, M.J. Stevens, R. P. Mirin, L. K. Shalm, M. A. Steinberg, Observing the Average Trajectories of Single Photons in a Two-Slit Interferometer, Science, 332, (2011) 1170-1173.

[51] C. Leforestier, R. H. Bisseling, C. Cerjan, M. D. Feit, R. Friesner, A. Guldberg, and N. Lipkin, A comparison of different propagation schemes for the time dependent Schrödinger equation. Journal of Computational Physics, 94(1) (1991) 59-80.

[52] N. Makri and W. H. Miller, Correct short time propagator for Feynman path integration by power series expansion in $\Delta t$, Chem. Phys. Lett. $1511-8,1988$. 
[53] N. Makri and W. H. Miller, Exponential power series expansion for the quantum time evolution operator. J. Chem. Phys. 90, (1989) 904-11.

[54] Mahler, D. H., Rozema, L., Fisher, K., Vermeyden, L., Resch, K. J., Wiseman, H. M., and Steinberg, A. (2016). Experimental nonlocal and surreal Bohmian trajectories. Science advances, 2(2), e1501466.

[55] A. Markowsky and N. Schopohl, Cold Bose atoms around the crossing of quantum waveguides. Phys. Rev. A 89 (2014) 013622.

[56] V. P. Maslov and M. V. Fedoriuk. Semi-classical approximation in quantum mechanics. Vol. 7. Springer Science \& Business Media, 2001.

[57] B. Misra, E. C. G. Sudarshan, The Zeno's paradox in quantum theory, J. Math. Phys. 18 (1977) 756-763.

[58] J. Morley, P. D. Edmunds and P. F. Barker, Measuring the weak value of the momentum in a double slit interferometer, J. Phys. Conference series, 701 (2016) 012030.

[59] N. F. Mott, The Wave Mechanics of $\alpha$-Ray Tracks. Proc. Royal Soc. of London A: Mathematical, Physical and Engineering Sciences. Vol. 126. No. 800. The Royal Society (1929) 79-84.

[60] E. Nelson, Topics in Dynamics I: Flows, Mathematical Notes, Princeton University Press, 1969.

[61] A. Peres, Zeno paradox in quantum theory, Am. J. Phys. 48 (1980) 931-2.

[62] C. Philippidis, C. Dewdney, B. J. and Hiley, Quantum Interference and the Quantum Potential, Nuovo Cimento 52B, (1979) 15-28.

[63] M. Reed and B. Simon. Methods of Modern Mathematical Physics I, II: Functional analysis. Fourier analysis, self-adjointness, Academic Press, New York (1975).

[64] A. Sanayei, A note on the short-time quantum propagator. arXiv preprint arXiv:1404.0506 (2014).

[65] L. S. Schulman, Techniques and applications of path integration, Wiley, New York, 1981.

[66] H. F. Trotter, On the product of semi-groups of operators. Proc. Amer. Math. Soc. 10 (1959) 545-51. 
[67] Wiseman, H.M., Directly observing momentum transfer in twin-slit Òwhich-wayÓ experiments, Phys. Letts. A 311, (2003) 285-291.

[68] R. E. Wyatt, Quantum dynamics with trajectories: introduction to quantum hydrodynamics. Vol. 28. Springer Science \& Business Media, 2006.

[69] Xiao-Song Ma, Herbst, T., Scheid, T., Daqing Wang, Kropatschek, S., Naylor, W., Wittmann, B., Mech, A., Kofler, J.,Anisimova, E., Makarov, V., Jennewein, T., Ursin, R. \& Anton Zeilinger, A., Quantum teleportation over 143 kilometres using active feed-forward, Nature, 489 (2012) 269-273.

[70] K. Yajima, Existence of Solutions for Schrödinger Evolution Equations, Commun. Math. Phys. 110, (1987), 415-426. 